\title{
Advanced Materials for a Sustainable, Clean Energy Future
}

\author{
Zhenguo "Gary" Yang
}

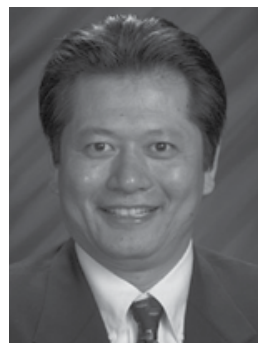

The current annual worldwide energy consumption stands at about 15 terawatts (TW, $\times 10^{12}$ watts). Approximately $80 \%$ of it is supplied from fossil

fuels: oil (34\%), coal (25\%), and natural gas $(21 \%)$. Biomass makes up $8 \%$ of the energy supply, while nuclear energy makes up $6.5 \%$, hydropower, $2 \%$, and other technologies such as wind and solar make up the rest. Even with aggressive conservation and new higher-efficiency technology development, worldwide energy demand is predicted to double to $30 \mathrm{TW}$ by 2050 and triple to 46 TW by the end of the century. Meanwhile oil and natural gas production is predicted to peak over the next few decades. Abundant coal reserves may maintain the current consumption level for a longer period of time than the oil and gas. However, burning the fossil fuels results in emissions of large amounts of greenhouse gases, particularly $\mathrm{CO}_{2}$, which are widely considered to be the primary contributor to global warming. Many countries, and even some states and cities in the United States, have adopted regulations to limit $\mathrm{CO}_{2}$ emissions. Also, carbon "trading" has emerged as a trend, giving benefits to low carbon-footprint industries, while making higher-emitting industries purchase carbon allowances. There has been an increasing number of countries and states adopting the trade and cap systems.

The environmental concerns and constraints of fossil fuels, combined with energy security worries, have spurred great interest and extensive R\&D efforts in developing clean coal technologies, renewable power generation from sources such as solar, and fuel-efficient technologies for transportation. Cheap and widely available, coal continues to be a major fuel in the future. But it has to be burned in a clean way in which carbon can be captured and sequestered underground. Solar radiation energy hitting the earth in one hour is enough for the energy consumption of the whole world for a year. Photovoltaic conversion of the solar energy figures to be a major approach to generate clean renewable energy. Transportation efficiency can be improved by the use of batteries in plug-in hybrid electrical vehicles. Potentially cleaner alternatives are fuel cells that burn hydrogen and emit only water vapor. But to have fuel cells in vehicles requires efficient on-board hydrogen storage. Among the most promising approaches is the solid-state storage of hydrogen. Advancement of the clean energy technologies relies on progress in advanced materials. Thus, five papers were invited to cover these areas in a JOM topic on Materials for a Clean Energy $\mathrm{Fu}$ ture.

Wei Liu et al. provide a short review of $\mathrm{CO}_{2}$ capture incentives, current separation processes, and research progress of various new technologies. Specifically, the paper focuses on separation of a gas mixture by distillation, absorption, adsorption, gas/solid reaction, membrane, electrochemical pump, hydrate formation, etc. It is concluded that the challenge lies in practical feasibility and ultimately the cost.

Writing about hydrogen storage, William Osborne et al. discuss storage capacity, thermodynamics, and kinetics of reversible solid-state storage that holds great promise for on-board applications. The authors point out that the key criteria for a successful solid-state reversible storage material are high storage capacity, suitable thermodynamic properties, and fast hydriding and dehydriding kinetics.

$\mathrm{K}$. Lee et al. discuss nanocrystalline $\mathrm{TiO}_{2}$ thin film electrodes for dye-sensitized solar cells (DSSC) that are considered as one of the most promising technologies with the potential of rendering solar energy as an economically attractive sustainable energy source. The paper describes the operation principles of DSSC and introduces current research efforts aiming for high efficiency and easy fabrication. In particular, the focus is on alternative methods of synthesis, coating, and processing the $\mathrm{TiO}_{2}$ nanocrystalline films as a critical operation in fabricating the DSSC.

In his paper, Neale Neelameggham discusses lightweight packaging of lithium-ion batteries for use in hybrid vehicles. The author suggests simpler battery pack designs based on electrorefining pot rooms using self-contained rectangular lithium-ion cells with air cooling inside of die-cast magnesium cell tanks.

The paper by Lanlin Zhang et al. discusses the promises and challenges of inorganic membranes. Supported inorganic membranes hold the promise of highly effective separation and purification, and stable operation in harsh environments. However, compared to organic membranes, large-scale production of inorganic membranes requires improvements in reproducibility and cost processes.

Zhenguo "Gary" Yang is with Pacific Northwest National Laboratory, 902 Battelle Blvd., Richland, WA 99354, and is the advisor to JOM from the Energy Conversion and Storage Committee of the Electronic, Magnetic \& Photonic Materials Division of TMS. 